\title{
Reaction-Diffusion Assisted Synthesis of Gold Nanoparticles: Route from the Spherical Nano-Sized Particles to Micrometer-Sized Plates
}

\author{
Szabolcs Farkas, Gábor Holló, Gábor Schuszter, Agota Deák, László Janovák, Viktória Hornok, \\ Masaki Itatani, Hideki Nabika, Dezső Horváth, Agota Tóth, and István Lagzi*
}

Cite This: J. Phys. Chem. C 2021, 125, 26116-26124

Read Online

ACCESS

Llll Metrics \& More

Article Recommendations

Supporting Information

ABSTRACT: The design of nanoparticles of desired sizes and shapes and 2D nanostructured materials is challenging and important due to their unique physical and chemical properties. One of the most common methods for the generation of metal nanoparticles is the wet synthetic route in which metal ions are reduced and the formed particles are stabilized in the liquid phase. Here we show a facile and powerful method to synthesize gold nanoparticles in a solid agarose gel utilizing the diffusion of reagents using the Turkevich method at room temperature. Our technique yields particles spatially separated by their sizes and shapes (spheres and plates) in the gel column. We have achieved 4 orders of magnitude difference in the sizes of the synthesized particles with a linearly increasing trend in the function of their spatial position. We have also generated micrometer-sized nanoplates with triangle, truncated triangle, or hexagon shapes attaining almost the length-to thickness ratio of 500, representing the magnificent power of the reaction-diffusion assisted synthesis.

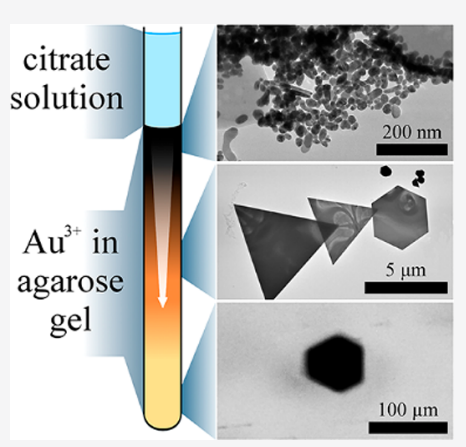

\section{INTRODUCTION}

The synthesis of metal nanoparticles (NPs) and their selfassembly into nanostructured materials have gained much attention in the past few decades due to their unique chemical, optical, magnetic, and electronic properties. ${ }^{1-5}$ They have been widely used in various applications ranging from catalysis $^{6-8}$ to nanomedicine ${ }^{9-13}$ to electronics. ${ }^{14-16}$ The most common technique to prepare metal NPs is the wet chemical synthesis method in a bulk phase, in which a metal salt and reducing as well as stabilizing (capping) agents have been used in the aqueous phase. ${ }^{17}$ The redox reaction produces metal atoms from metal ions, and they form nuclei (nucleation step), which can grow producing nanometer-sized particles (growth step). ${ }^{18,19}$ The stabilizing agent adsorbing onto the surface of the formed particles inhibits further particle growth and produces a stable dispersion of NPs. ${ }^{20}$ The oldest wet synthesis route to generate spherical gold nanoparticles (AuNPs) is the Turkevich method. ${ }^{21}$ It is based on the reduction of a gold salt by citrate at elevated temperature (usually $T>70{ }^{\circ} \mathrm{C}$ ), where citrate plays a role as a reducing and stabilizing agent as well. This one-step method is simple and highly reproducible; however, it has several drawbacks, namely, the generated particles are mostly spherical with the size ranging between 10 and $30 \mathrm{~nm}$. To synthesize larger (or smaller, sub-10 nm) spherical particles and other shapes (rod, triangle, truncated triangle, and hexagon in $2 \mathrm{D}$ and polyhedral shapes-decahedron, icosahedron, and tetrahedron-in 3D) additional growing steps (seed-mediated growth) and/or the usage of additives (e.g., surfactants, macromolecules) are needed. $^{2-33}$ These additives adsorbing onto the specific crystal plain inhibit the further crystal growth in this direction, thus the growth occurs along preferential crystal plains producing various nonspherical structures. Surfactants such as cetyltrimethylammonium bromide (CTAB) and cetyltrimethylammonium chloride (CTAC) adsorb on the lowest energy $\{111\}$ facets and suppress their growth, which in turn directs and promotes the evolution of nanoplates. ${ }^{24,30}$ Usually to synthesize nanoplates with a size greater than 100 $\mathrm{nm}$, seed-mediated growth methods have been used. ${ }^{34,35}$ Besides, one-step photochemical reduction methods were also proposed in the literature. ${ }^{36,37}$ Generating both spherical nanoparticles and micrometer-sized nanoplates in a predictable size in a one-step method is challenging. Wet bulk methods lack the ability to create NPs of various sizes and shapes (particle libraries) by using fixed conditions without repetition with a new set of experimental parameters.

Here we show a facile and powerful method to synthesize AuNPs with various sizes and shapes in a single experiment at room temperature, based on the Turkevich method, utilizing the temporal change in the diffusive fluxes of the reagents in a solid hydrogel matrix in a Liesegang-type experimental setup. ${ }^{38}$ In this setup, one reagent is homogeneously distributed in a solid hydrogel (inner reagent) while the other reagent is placed

Received: September 24, 2021

Revised: November 5, 2021

Published: November 18, 2021 
on top of the gel column (outer reagent). The process is governed by the reaction between the reagents and the diffusion front of the outer reagent because its concentration is 1 or 2 orders of magnitude greater than that of the inner one. The obtained library of nanoparticles contains spatially separated spherical AuNPs with the size of 20-30 nm and gold nanoplates (triangle, truncated triangle, and hexagon) of sizes ranging from a few hundred nanometers to $70 \mu \mathrm{m}$.

\section{EXPERIMENTAL SECTION}

\subsection{Synthesis of Gold Nanoparticles in the Agarose} Hydrogel. The agarose powder (Sigma-Aldrich, Type I) was dissolved in distilled water, and the mixture was continuously stirred by using a magnetic stirrer $(500 \mathrm{rpm})$ at $90{ }^{\circ} \mathrm{C}$ until complete dissolution of the agarose $(\sim 10-15 \mathrm{~min})$. Then the heating was switched off, while maintaining the continuous stirring. When its temperature decreased to $60{ }^{\circ} \mathrm{C}$, the room temperature $\left(22.0 \pm 0.5{ }^{\circ} \mathrm{C}\right)$ solution of gold(III) chloride trihydrate $\left(\mathrm{HAuCl}_{4} \cdot 3 \mathrm{H}_{2} \mathrm{O}\right.$, Sigma-Aldrich) of various concentrations was added to the agarose solution, and after complete homogenization, the solution was gently poured into test tubes (inner diameter and length of $6.0 \mathrm{~mm}$ and $25.0 \mathrm{~cm}$, respectively), creating a gel column of $15 \mathrm{~cm}$ with the agarose concentration of $0.1 \% \mathrm{~m} / \mathrm{v}$. The gelation process was accomplished in a refrigerator at $\sim 5{ }^{\circ} \mathrm{C}$. After gelation $(\sim 24$ $\mathrm{h}$ ), the gel column was allowed to rest for $1 \mathrm{~h}$ at room temperature, and then the citrate solution (sodium citrate dihydrate, $\mathrm{HOC}(\mathrm{COONa})\left(\mathrm{CH}_{2} \mathrm{COONa}\right)_{2} \cdot 2 \mathrm{H}_{2} \mathrm{O}$, Sigma-Aldrich) was gently poured on top of the hydrogel column to create a liquid layer of $10 \mathrm{~cm}$ above the solid gel column. All experiments were performed at room temperature $(22.0 \pm 0.5$ ${ }^{\circ} \mathrm{C}$ ) usually for 4 weeks (see the Supporting Information, Figure S1).

2.2. Transmission Electron Microscopy (TEM), Scanning Electron Microscopy (SEM), and Atomic Force Microscopy (AFM) Measurements. To study the morphology of the nanoparticles, transmission electron microscopy (TEM) measurements were performed by using a FEI Tecnai G2 20 X-TWIN microscope with a tungsten cathode operated at $200 \mathrm{kV}$. For TEM measurements, $10 \mu \mathrm{L}$ of AuNP dispersion was dropped on a grid (carbon film with 200 mesh copper grids, CF200-Cu, Electron Microscopy Sciences, USA) and dried at room temperature. The structures of the synthesized AuNPs were examined by scanning electron microscopy (SEM, Hitachi S-4700 microscope), applying a secondary electron detector and $10 \mathrm{kV}$ acceleration voltage. Energy dispersive X-ray spectra were measured by using the Röntec EDX detector at $15 \mathrm{keV}$. AuNPs were investigated by using a Multimode Nanoscope IIIa atomic force microscope (AFM, Digital Instruments, USA). The images were acquired in tapping mode in air at room temperature by using a Si tip cantilever (Veeco Nanoprobe Tips RTESPA model) with a resonance frequency of $275-300 \mathrm{kHz}$. Height- and amplitudemode images were recorded simultaneously with $0.8 \mathrm{~Hz}$ scan rate. Processing and analysis of the images were performed by using the off-line software Nanoscope V614r1. The samples for AFM were prepared by depositing a dilute dispersion of AuNPs on glass holder and were left to dry at room temperature.

2.3. Isolation of the Produced Gold Nanoparticles from the Agarose Matrix. The AuNPs regions of the tubes were divided into consecutive zones of $0.5 \mathrm{~cm}$ thickness. The regions were extracted and washed three times with $1 \mathrm{~mL}$ of hot DMF ( $N, N$-dimethylformamide, Merck) and centrifuged $(20 \mathrm{~min}, 14500 \mathrm{rpm})$ to dissolve the gels and then redispersed in $1 \mathrm{~mL}$ of ultrapure water.

2.4. Bulk Synthesis of Gold Nanoparticles Using Agarose. The agarose powder (Sigma-Aldrich, Type I) was dissolved in distilled water, and the mixture was continuously stirred by using a magnetic stirrer $(500 \mathrm{rpm})$ at $90{ }^{\circ} \mathrm{C}$ until complete dissolution of the agarose $(\sim 10-15 \mathrm{~min})$. Then the heating was switched off, while maintaining the continuous stirring. When its temperature decreased to $60{ }^{\circ} \mathrm{C}$, the room temperature $\left(22.0 \pm 0.5{ }^{\circ} \mathrm{C}\right)$ solutions of gold(III) chloride trihydrate $\left(\mathrm{HAuCl}_{4} \cdot 3 \mathrm{H}_{2} \mathrm{O}\right.$, Sigma-Aldrich $)$ and sodium citrate dihydrate $\left(\mathrm{HOC}(\mathrm{COONa})\left(\mathrm{CH}_{2} \mathrm{COONa}\right)_{2} \cdot 2 \mathrm{H}_{2} \mathrm{O}\right.$, Sigma-Aldrich) were added to the agarose solution. After complete homogenization, the solution was gently transferred into glass vials, and then those vials were sealed. The bulk synthesis was performed at room temperature $\left(22.0 \pm 0.5{ }^{\circ} \mathrm{C}\right)$ for 1 week.

\section{RESULTS AND DISCUSSION}

In a typical experiment, the solid agarose gel column was prepared in test tubes containing the prescribed concentration of gold salt. After the gelation $(\sim 24 \mathrm{~h})$, a citrate solution was gently poured on top of the hydrogel column with the length of $15 \mathrm{~cm}$ (Figure S1). The concentration of the citrate was set to be greater than that of the gold salt because excess of the citrate is needed in the synthesis of AuNPs, and second, it ensured that the formation of the particles occurred also at the bottom part of the gel column. The agarose itself, as a polysaccharide, can reduce $\mathrm{Au}^{3+}$ to $\mathrm{Au}^{0}$ and produce AuNPs at elevated temperature $\left(\sim 70-90{ }^{\circ} \mathrm{C}\right){ }^{39}$ Therefore, the agarose concentration was reduced to the value at which no formation of AuNPs was observed at room temperature, and the gel matrix was solid enough to provide a medium for convectionfree mass transport of the reagents realized solely by diffusion. Additionally, the gel matrix prevents the sedimentation of the formed particles. To investigate the effect of the initial concentration of the reagents on the characteristics of the produced particles and macroscopic pattern structures, a set of concentration pairs of $\mathrm{Au}(\mathrm{III})$ and citrate was tested (Figure S2).

The citrate layered above the hydrogel column diffused in the gel matrix containing $\mathrm{Au}(\mathrm{III})$. After 1 day of the process, a dark red color appeared near the liquid-gel interface (junction point of the reagents), indicating the formation of AuNPs due to their prominent surface plasmon resonance (SPR). The time of appearance of the AuNPs was significantly greater than in the syntheses at elevated temperature $\left(\sim 70-100{ }^{\circ} \mathrm{C}\right)$ since the rate of the reduction is considerably slower at room temperature than at elevated temperature. In time, as the diffusion front of the citrate moved deeper into the gel matrix, it caused the continuous formation of the AuNPs along the gel column. Figure la shows a control experiment indicating no formation of particles induced solely by the agarose gel matrix. In the presence of citrate, however, reduction of $\mathrm{Au}(\mathrm{III})$ occurred, producing AuNPs ordered in the macroscopic patterns (Figure $1 \mathrm{~b}, \mathrm{c}$ ). The pattern is characterized by a continuous structure at a high salt concentration $\left(\left[\mathrm{Au}^{3+}\right]=10\right.$ $\mathrm{mM}$ ); however, at lower concentrations periodic precipitation structures appeared consisting of several well-separated bands of AuNPs formed from a redox reaction. Interestingly, this phenomenon is vividly manifested in the case of $\left[\mathrm{Au}^{3+}\right]=5$ $\mathrm{mM}$ and [citrate] $=0.1 \mathrm{M}$ (Figure S3). Similar patterns have been observed in diffusion/coprecipitation systems in which a 


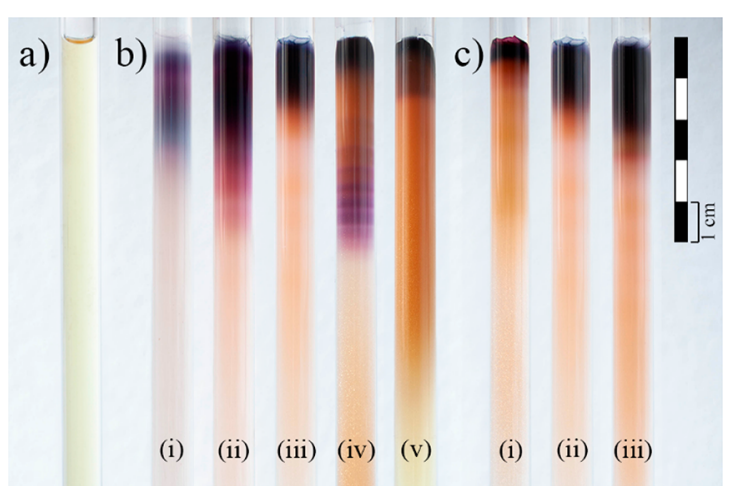

Figure 1. Patterns generated by the synthesized AuNPs in an agarose gel $(0.1 \% \mathrm{~m} / \mathrm{v})$ column containing $\mathrm{Au}(\mathrm{III})$ ions after 4 weeks. The solution of citrate was layered onto the gel column. (a) Control experiment in the absence of the citrate solution, the agarose gel contained gold salt with $\left[\mathrm{Au}^{3+}\right]=10 \mathrm{mM}$; no formation of AuNPs. (b) Patterns in the agarose gel containing $\mathrm{Au}(\mathrm{III})$ ions with the concentration of (i) $\left[\mathrm{Au}^{3+}\right]=0.5 \mathrm{mM}$, (ii) $\left[\mathrm{Au}^{3+}\right]=1 \mathrm{mM}$, (iii) $\left[\mathrm{Au}^{3+}\right]=2 \mathrm{mM}$, (iv) $\left[\mathrm{Au}^{3+}\right]=5 \mathrm{mM}$, and (v) $\left[\mathrm{Au}^{3+}\right]=10 \mathrm{mM}$ at fixed citrate concentration $([$ citrate $]=0.1 \mathrm{M})$. (c) Patterns at fixed $\mathrm{Au}(\mathrm{III})$ concentration in the agarose gel $\left(\left[\mathrm{Au}^{3+}\right]=2 \mathrm{mM}\right)$, while the concentration of the citrate was varied: (i) $[$ citrate $]=0.01 \mathrm{M}$, (ii) $[$ citrate $]=0.1 \mathrm{M}$, and (iii) $[$ citrate $]=1 \mathrm{M}$.

reaction of two water-soluble salts forming insoluble inorganic precipitate coupled to the diffusion of the reagents in a solid gel matrix leads to spatially periodic precipitation (Liesegang phenomenon). ${ }^{38}$ It should be noted that similar behavior was observed in the formation of metal-organic frameworks in a gelled medium. ${ }^{40}$ The remarkable feature of our pattern generated by a redox reaction is that the distance between the two consecutive bands decreases along the gel column (Figure S4). This is a very rarely observed revert periodic precipitation as the usual trend is an increase of the distance between the two consecutive bands in the band number. ${ }^{41}$ Revert periodic precipitation occurs when the excess of invading ions (diffusing from outside, e.g., silver ions) can be also efficiently adsorbed on the surface of the formed particles (e.g., silver halides) forming a charged double layer, thus electrostatically stabilizing them and preventing further aggregation to form a precipitate. ${ }^{42-44}$ Farther from the liquid-gel interface, the excess of the stabilizing compound decreases due to the diffusive nature of the front involving a decrease of the stabilization capacity, thus facilitating the coagulation. This results in the decrease in the distance between the two consecutive bands as the band number increases. The formation of revert periodic precipitation in our system can be explained in a similar manner since the citrate is the particle not only producing agent but also stabilizing the formed AuNPs.

To characterize quantitatively the formed particles by size, shape, and composition, transmission electron microscopy (TEM), scanning electron microscopy (SEM) with energy dispersive X-ray analysis (EDX), atomic force microscopy (AFM), and optical microscopy were deployed. After 1 month the gel was removed from the glass tube and cut into several segments. The generated AuNPs were extracted from the gel and transferred to the sample holder. Figure 2 shows the characteristics of the formed AuNPs at various distances from the liquid-gel interface. Near the interface $(0-0.5 \mathrm{~cm})$, only spherical particles of the size of $\sim 20-30 \mathrm{~nm}$ formed; ${ }^{21}$ farther from the liquid-gel interface, the size of the AuNPs became greater and nanoplates (triangles, truncated triangles, and hexagons) started to appear in the samples. With an increasing distance from the liquid-gel interface, the probability of the formation of nanoplates with various shapes increased. This trend can be observed in other initial conditions of the reagents as well (Figures S5 and S6).

On the basis of the detailed analysis of the particle size along the gel column in the case of $\left[\mathrm{Au}^{3+}\right]=2 \mathrm{mM}$ and [citrate] = $0.1 \mathrm{M}$, we observed a linear trend with an increasing polydispersity of the samples (Figure $3 a$ and Figure S7). A similar tendency was found in other reaction-diffusion systems having silver dichromate precipitate and zeolitic imidazolate framework-8 (ZIF-8) crystals formed from the coprecipitation of silver and dichromate ions and zinc ions and 2-methylimidazole organic linkers, respectively. ${ }^{40,45,46}$ The increasing size of the particles can be explained by the following arguments: the nucleation rate and its dependence on the supersaturation. Near the liquid-gel interface, the concentration of the citrate is the highest, thus providing the highest supersaturation and nucleation rate of reduced gold atoms generating a high number of nuclei. Therefore, here the reagents are mostly consumed in the nucleation step, growth is hindered, and thus small particles form. In addition to this, the citrate adsorbs on the particle surface and suppresses further growth as well. Farther from the interface the local citrate

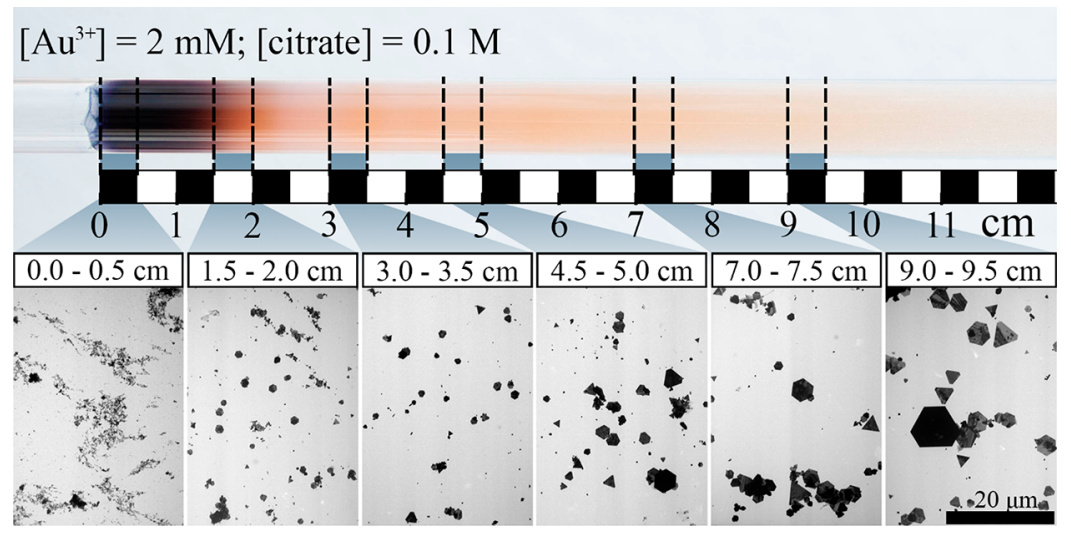

Figure 2. Synthesized AuNPs of various sizes and shapes in a reaction-diffusion process in an agarose hydrogel $(0.1 \% \mathrm{~m} / \mathrm{v})$ containing homogeneously distributed gold salt $\left(\left[\mathrm{Au}^{3+}\right]=2 \mathrm{mM}\right)$, the citrate $([$ citrate $]=0.1 \mathrm{M})$ diffused from the outside (from the liquid phase placed on top of the gel column). 

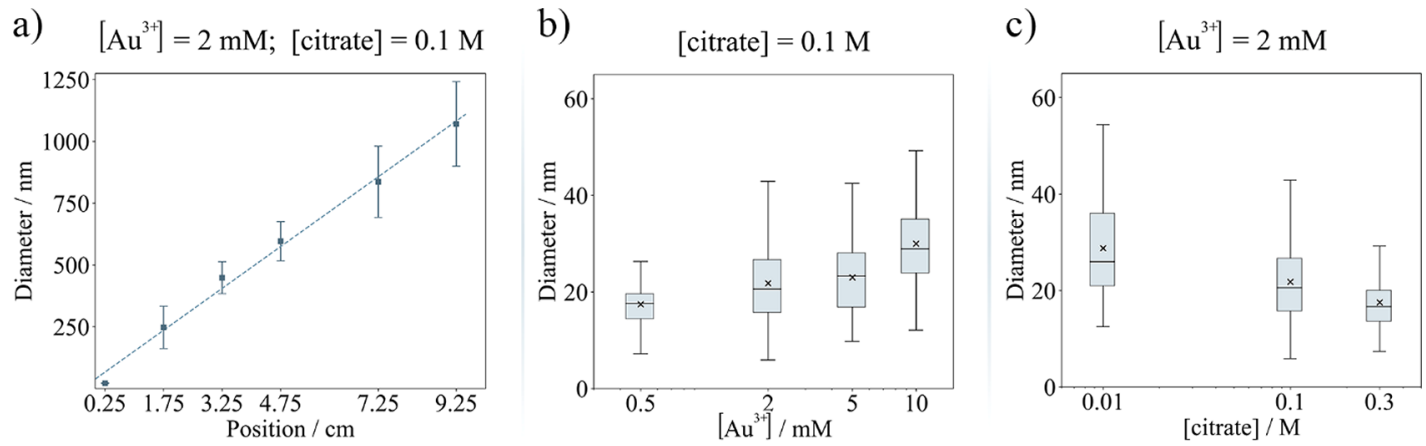

Figure 3. (a) Dependence of the average size of the synthesized AuNPs on their position in the hydrogel matrix measured from the liquid-gel interface. (b) Dependence of the average size of AuNPs generated near the liquid-gel interface $(0-0.5 \mathrm{~cm}$ gel segment measured form the liquidgel interface) on the gold salt concentration at fixed citrate concentration ([citrate] $=0.1 \mathrm{M})$ and $(\mathrm{c})$ on the citrate concentration at fixed gold salt concentration $\left(\left[\mathrm{Au}^{3+}\right]=2 \mathrm{mM}\right)$.

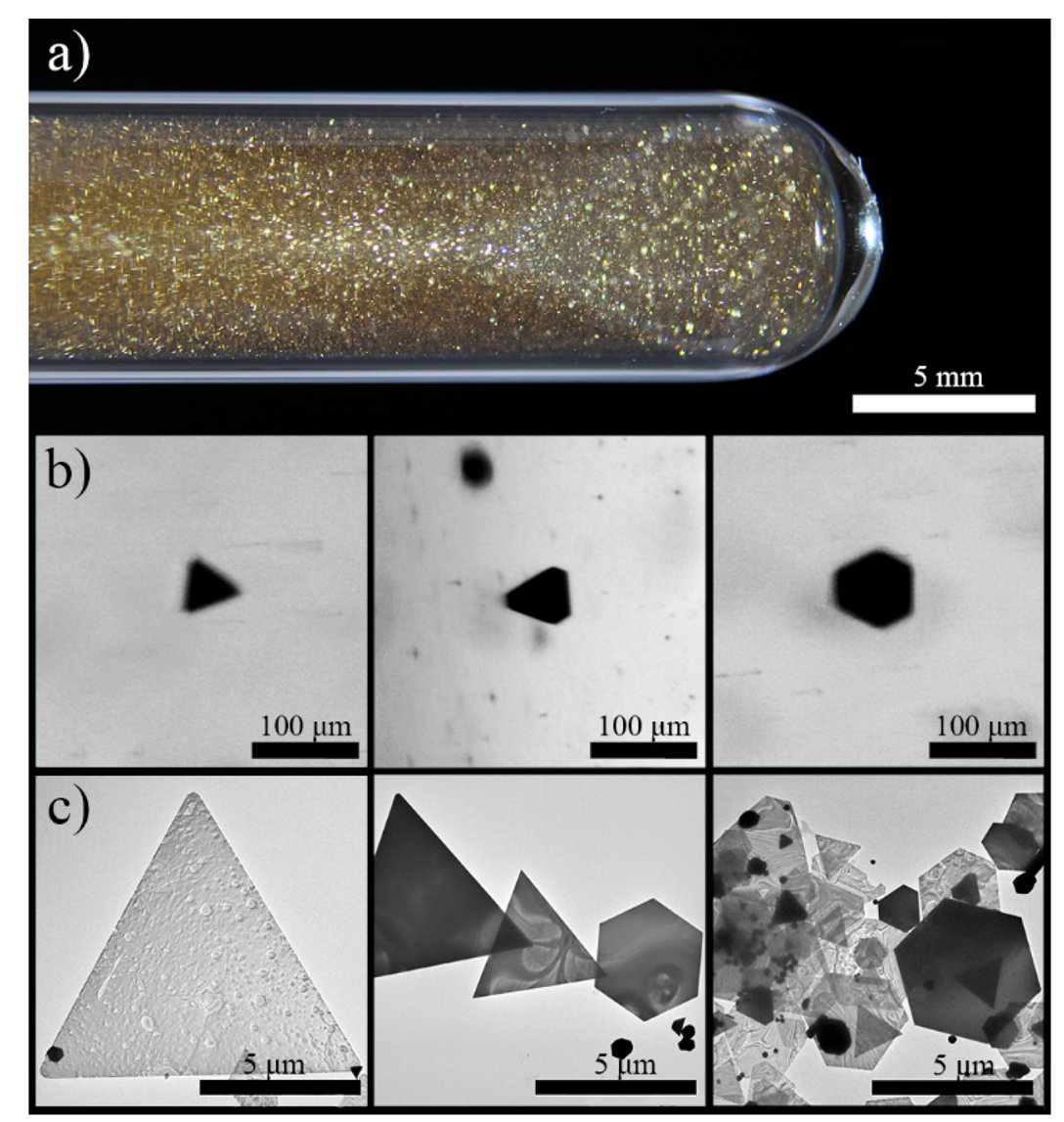

Figure 4. (a) Optical photograph of the agarose gel in a glass test tube using reflected illumination; the appearance of the bulk optical property of the gold can be vividly seen. (b) Optical micrographs of the generated nanoplates at the bottom part of the gel column (10-15 cm measured from the liquid-gel interface) by using transmitted illumination: triangle, truncated triangle, and hexagon. (c) TEM micrographs of the nanoplates extracted from the region of $\sim 4-8 \mathrm{~cm}$ measured from the liquid-gel interface.

concentration is smaller due to the diffusive nature of the citrate front. The supersaturation and nucleation rate decrease, creating a smaller number of nuclei, and therefore the further particle growth can be "fueled" by the counterdiffusion of the reagents; i.e., the backward diffusion of $\mathrm{Au}(\mathrm{III})$ ions from the ahead region of the forward propagating citrate-driven reaction-diffusion front. Note that near the interface the backward diffusion of $\mathrm{Au}$ (III) ions cannot occur because-due to the big initial concentration difference-the fast traveling citrate front consumes all $\mathrm{Au}(\mathrm{III})$ ions in the wake of the front by the redox reaction generating nuclei.
EDX experiments confirmed the formation of gold particles with shapes of spheres and plates in the hydrogel matrix (Figure S8). We investigated the average sizes and size distribution of the AuNPs formed near the liquid-gel interface $(0-0.5 \mathrm{~cm})$ at various initial concentrations of the reagents. Figure $S 9$ represents the corresponding size distributions, and Figure $3 b, c$ shows the dependence of the average particle size on the concentrations of gold salt and citrate. It can be concluded that the average size ranged between 20 and $30 \mathrm{~nm}$, which is a size range that can be obtained in the bulk Turkevich method. ${ }^{21}$ Higher gold salt concentration at fixed 
citrate concentration resulted in larger AuNPs (Figure 3b), and an increased citrate concentration at fixed concentration of $\mathrm{Au}(\mathrm{III})$ generated smaller particles (Figure 3c). This observation is in good accordance with the general trend observed in bulk synthesis of citrate-stabilized AuNPs; namely, higher ratios of the citrate concentration to the $\mathrm{Au}$ (III) concentration result in smaller AuNPs because citrate is a stabilizing agent, and hence it prevents further crystal growth. ${ }^{47-49}$ A similar trend can be noticed in the final sizes of nanoplates. Lower citrate concentration allowed the formation of larger nanoplates at a fixed concentration of the $\mathrm{Au}(\mathrm{III})$ (Figure S6).

The biggest nanoplates found in the samples were $\sim 70 \mu \mathrm{m}$ in their sizes (at the bottom of the gel matrix, 10-15 cm) (Figure 4), but the average size of the plates ranged between 10 and $30 \mu \mathrm{m}$. Interestingly, there is almost 4 orders of magnitude difference between the smallest and biggest AuNPs in one sample. Because of the presence of micrometer-sized particles, the sample lost the nanoscale optical property, namely the SPR, and became yellow, corresponding to the optical property of the bulk gold (Figure 4). The thickness of the nanoplates ranged between a few tens of nanometers $(\sim 50$ $\mathrm{nm}$ ) to a few hundred nanometers based on SEM and AFM measurements (Figure 5, Figures S10 and S11). The thicknessto-length ratio in some cases approached 500, which characterizes a unique nanothick $2 \mathrm{D}$ gold sheet (Figure 5 and Figures S11).

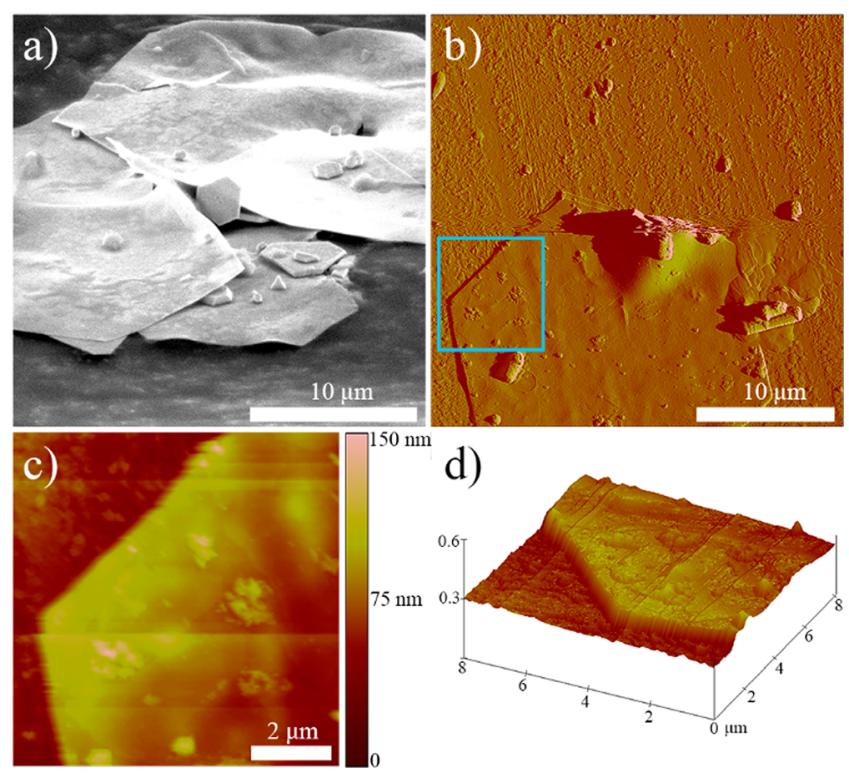

Figure 5. (a) SEM and (b-d) AFM micrographs of the nanoplates extracted from the region of $10-15 \mathrm{~cm}$ measured from the liquid-gel interface. (c, d) An enlarged part of a nanoplate indicated by a blue box in (b).

We exclude the photochemical route to contribute to the formation of AuNPs (as hypothesized in the paper by Pienpinijtham et al. on using starch-mediated photochemical reduction to generate gold nanoplates ${ }^{37}$ ) because no particles formed in the agarose gel in the absence of citrate even if the highest concentration of the gold salt was used in the experiments (10 mM, Figure 1a). Additionally, we performed the formation of AuNPs in the gel matrix in the absence (dark) and presence of the illumination and found no difference in the samples (Figure S12).

We hypothesize that the mechanism of the formation of the nanoplates is the following: near the interface the local concentration of the citrate is high, causing fast nucleation and efficient stabilization creating small spherical particles. Farther from the liquid-gel interface, the nucleation rate slows down. The polysaccharide (the component of the agarose gel) adsorbs to the lowest surface energy $\{111\}$ facets of the formed particles and promotes the slow growth of plate-like particles. Additionally, the slow backward diffusion (compared to the nucleation event) of gold ions from the neighboring ahead domain helps the slow particle growth. The porous gel matrix prevents the sedimentation and aggregation of nanoplates by van der Waals (vdW) forces, thus creating an optimal condition for the generation of micrometer-sized gold plates. The crystal growth to nucleation event ratio is higher at larger distances which is also reflected by the linearly increasing particle size (Figure 3a).

It should be noted that at the beginning of the process (closer to the liquid-gel interface) small spherical particles were formed, which might self-assembled into a banded pattern. However, at the later stage (farther from the liquidgel interface), larger particles grew to have less ability to diffuse due to their size and reaching the size of the pore of the agarose gel (approximately a few hundred nanometers). ${ }^{50}$ Therefore, they could not generate zones and produced a homogeneous distribution of the nanoplates in the gel.

To highlight the power of the synthesis of AuNPs in the solid gel driven by reaction and diffusion, we performed bulk experiments with identical initial concentrations to that of the diffusion experiments. In these setups, the process was completed within a few hours, forming dominantly black precipitate, and in a few cases, the samples had red, purple, and blue colors consisting of $\sim 20-30 \mathrm{~nm}$ spherical AuNPs without the formation of nanoplates (Figure S13).

To illustrate the formation of AuNPs in a hydrogel, we have developed a reaction-diffusion (RD) model incorporating diffusion, nucleation, and particle growth. The mechanism of the formation of nanoparticles is the following:

$$
\begin{aligned}
& \mathrm{A}+\mathrm{B} \rightarrow \mathrm{C} \\
& \mathrm{C}+\mathrm{C} \rightarrow \mathrm{D} \\
& \mathrm{C}+\mathrm{D} \rightarrow \mathrm{D} \\
& \mathrm{A}+\mathrm{B}+\mathrm{C} \rightarrow \mathrm{D}
\end{aligned}
$$

where $\mathrm{A}$ and $\mathrm{B}$ denote the reagents, citrate and $\mathrm{Au}^{3+}$, respectively. $\mathrm{C}$ and $\mathrm{D}$ are the nuclei and formed NPs, respectively. Equation 1 represents the formation of the nuclei. The other steps show the successive transformations of nuclei into NPs by nuclei-nuclei (eq 2), nuclei-NP aggregation (eq 3 ), and heterogeneous particle growth (eq 4). The system can be described mathematically in one dimension (along the gel column) by the following set of partial differential equations:

$$
\begin{aligned}
& \frac{\partial a}{\partial t}=D_{\mathrm{A}} \frac{\partial^{2} a}{\partial x^{2}}-k_{1} a b-k_{4} a b c \\
& \frac{\partial b}{\partial t}=D_{\mathrm{B}} \frac{\partial^{2} b}{\partial x^{2}}-k_{1} a b-k_{4} a b c
\end{aligned}
$$




$$
\frac{\partial c}{\partial t}=D_{\mathrm{C}} \frac{\partial^{2} c}{\partial x^{2}}+k_{1} a b-2 k_{2} c^{2} \Theta\left(c-c^{*}\right)-k_{3} c d-k_{4} a b c
$$

$$
\frac{\partial d}{\partial t}=k_{2} c^{2} \Theta\left(c-c^{*}\right)+k_{4} a b c
$$

where $a, b, c$, and $d$ are the concentrations of $\mathrm{A}, \mathrm{B}, \mathrm{C}$, and $\mathrm{D}$, respectively. We introduced a quantity $\left(n_{d}\right)$ describing the concentration of nuclei in the formed NPs (D); thus, the number of the nuclei embedded in the NPs is $n=n_{d} / d$. We can derive two important quantities characterizing the NPs, namely the radius $(r)$ and the surface area $(A)$ of the particles, which are proportional to $n^{1 / 3}$ and $n^{2 / 3}$, respectively. We used constant reaction rate coefficients for reactions represented by eqs 1 and 2 (for details see the Supporting Information), but the reaction rate for the nuclei-NP aggregation (eq 3) was proportional to the surface area of the particles $\left(k_{3}=10^{-5}(1+\right.$ $\left.\left.10 n^{2 / 3}\right)(\mathrm{M} \mathrm{s})^{-1}\right) . D_{\mathrm{A}}, D_{\mathrm{B}}$, and $D_{\mathrm{C}}$ are the diffusion coefficients of the reagents and the nuclei in the gel. We assumed that the formed NPs (D) do not diffuse in the gel matrix. $\Theta$ is the Heaviside step function, and $c^{*}$ is the threshold concentration of the formation of $\mathrm{D}$ by nuclei-nuclei aggregation. We arbitrary set $c^{*}$ as a function of $\mathrm{Au}(\mathrm{III})$ concentration $(b)$ in the form of $c^{*}=c_{0}^{*}-b$, where $c_{0}^{*}$ is a constant because it reflects the fact that at greater and lower concentrations of $\mathrm{Au}(\mathrm{III})$ the same amount of citrate can stabilize less (lower aggregation threshold) and more efficiently (greater aggregation threshold) of the formed AuNPs, respectively.

It should be noted that the reaction $\mathrm{C}+\mathrm{C} \rightarrow \mathrm{D}$ (eq 2) has a threshold concentration, where nuclei aggregate to the nanoparticle. Citrate (A) is not only a reducing but also a stabilizing (capping) agent of the NPs. We hypothesized that the nuclei are stabilized as well by citrate, and their aggregation occurs only above a certain threshold concentration (eq 2). In eqs 3 and 4, nuclei grow until they create a nanoparticle, which processes are not hindered by the stabilizing effect of the citrate. The diffusion coefficient of the reagents $\left(D_{\mathrm{A}}=D_{\mathrm{B}}=\right.$ $\left.10^{-9} \mathrm{~m}^{2} \mathrm{~s}^{-1}\right)$ is a typical value for small hydrated ions, while the diffusion coefficient of the nuclei was chosen to be smaller (2 $\times 10^{-10} \mathrm{~m}^{2} \mathrm{~s}^{-1}$ ) since their size exceeds the size of small hydrated ions. The kinetic constants were chosen arbitrarily; $k_{1}$ is the largest rate constant (eq 1) since the reaction rate of the reduction could be much greater than that of the aggregation of two nuclei. The aggregation is a slower process because of the reduced mobility of the nuclei and not every collision results in aggregation since these nuclei are electrostatically stabilized by citrate. The rate coefficient of the reaction $\mathrm{C}+\mathrm{D}$ $\rightarrow \mathrm{D}$ was proportional to the surface of the nanoparticles because larger nanoparticles have a larger cross section. The reaction rate, in this case, was significantly lower than the chemical rate of the reduction (eq 1). With the typical set of concentrations used in the simulation, the slowest process was reaction $\mathrm{A}+\mathrm{B}+\mathrm{C} \rightarrow \mathrm{D}$, which is a third-order reaction. The initial and boundary conditions were set to represent the experimental conditions, namely, $a(t=0, x)=c(t=0, x)=d(t$ $=0, x)=n_{d}(t=0, x)=0, b(t=0, x)=b_{0}$ (where $b_{0}$ is the initial concentration of $B$ ), and we used no-flux boundary conditions at the end of the domain and Dirichlet boundary conditions at the liquid-gel interface for all species. $a(t, x=0)$ $=1 \mathrm{M}$, and $b(t, x=0)=c(t, x=0)=d(t, x=0)=n_{d}(t, x=$ $0)$. The details of the numerical model and the simulations can be found in the Supporting Information.
The simulation results of the reaction-diffusion model provided a qualitative agreement with the results observed in experiments (Figure 6, Figures S14 and S15). At high gold salt a)

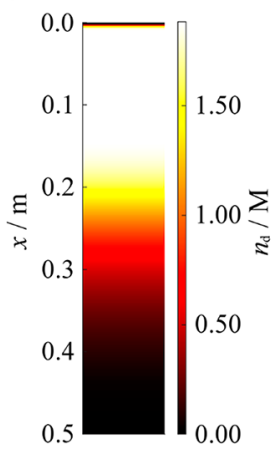

b)

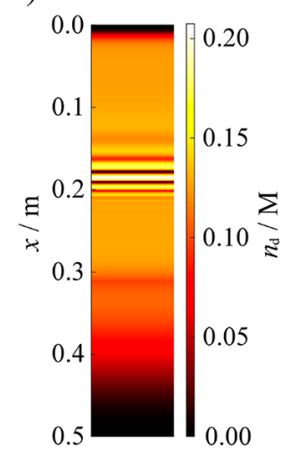

Figure 6. Results of the numerical simulations showing spatial distribution of the concentration of nuclei in particles $\left(n_{d}\right)$ : (a) $b_{0}=1$ and (b) $b_{0}=0.1$.

concentration, we observed a continuous zone of the formed AuNPs. However, when the concentration of $\mathrm{Au}(\mathrm{III})$ was decreased, the generated AuNPs self-assembled into periodic structures (Figure 1b). The size of the formed particles in the model is linearly proportional to their position measured from the liquid-gel interface, which is similar to the experiments (Figures S14 and S15). It should be noted that our model cannot capture the formation of nanoplates since we considered only spherical particles in the model; however, it shows that coupling diffusion to the reaction can provide conditions differing from the bulk setup and generate larger particles along the diffusion column. Interestingly, the numerical model reproduced the general trend in the bulk nucleation and growth scenario; namely, at low concentrations of the reagents small number of particles was formed with larger size. However, at greater concentrations of the reagents (higher supersaturation) a huge number of particles was formed with smaller size (Figure S16).

\section{CONCLUSIONS}

In summary, we have demonstrated a general and facile method to synthesize AuNPs of various sizes (from $\sim 10 \mathrm{~nm}$ to $70 \mu \mathrm{m}$ ) and shapes (spherical, triangular, truncated triangular, and hexagonal) separated spatially in a hydrogel matrix by their average size utilizing the effect of the diffusion of the reagents on the particle growth. The classical method to generate AuNPs was extended spatially utilizing the interplay between the chemical processes (including redox reaction, nucleation, and particle growth) and diffusion of the reagents in a solid hydrogel matrix. The main strength of our approach is that almost a 4 orders of magnitude difference in the sizes of the synthesized particles can be achieved in a single experiment with a linearly increasing average size in the function of their position in the gel matrix measured from the liquid-gel interface. The dynamics of the formation of particles is determined by the diffusion front of the reducing agent; its mass flux is one of the main factors governing the number of nuclei. These nuclei can grow, and their growth rate and final size are predominantly driven by the rate of formation of the nuclei and local influx of the $\mathrm{Au}(\mathrm{III})$ and formed nuclei from the neighborhood. We have shown that the nucleation and 
particle growth can be controlled by the fluxes of the citrate (invading from outside) and the $\mathrm{Au}(\mathrm{III})$ ions and nuclei through the local velocity of the diffusion front. Because the fluxes of the reagents and the nuclei strongly depend on the position of the reaction front, controlling these fluxes either spatially or temporally can provide flexible control in the synthesis of NPs.

One of the main advantages of our approach is that various shapes of AuNPs can be synthesized in a single process at room temperature utilizing the coupling of reaction and diffusion of the reagents. In addition to this, we have shown that a redox reaction extended in space through diffusion can generate periodic precipitation consisting of AuNPs of different sizes and shapes. Our findings highlight the universality of the periodic precipitation which has been observed so far in coprecipitating systems involving the reaction of ions forming a chemical precipitate. The most striking feature of our setup is that we could synthesize nanothick micrometer-sized plates of various shapes, where the length-to thickness ratio approached 500. This finding highlights the advantages of the synthesis utilizing diffusion in a solid gel matrix. Namely, farther from the liquid-gel interface, the diffusion slowly supplies the reagents generating slow and continuous particle growth. In addition to this, the gel matrix traps the formed plates and therefore prevents the aggregation of these thin micrometer-sized plates by attractive $\mathrm{vdW}$ interaction. In bulk liquid synthesis methods, these individual micrometer-sized plates cannot be preserved because these gold plates would stick together irreversibly due to the enormous vdW forces acting between two micrometer-sized plates. A future challenge would be to grow these unique gold structures on a $2 \mathrm{D}$ template and use them in microelectronics. This conductive material could have unique physical and chemical properties similar to graphene since the thickness is in nanoscale while the characteristic length belongs to micrometer size domain having bulk material properties. ${ }^{51}$ Moreover, we believe that controlling diffusion over chemical reactions can be exploited in various applications in material design and engineering.

\section{ASSOCIATED CONTENT}

\section{(3) Supporting Information}

The Supporting Information is available free of charge at https://pubs.acs.org/doi/10.1021/acs.jpcc.1c08404.

Numerical model description, experimental setup, formation of revert periodic precipitation, TEM micrographs of the synthesized AuNPs, size distribution diagrams and EDX elemental maps of the synthesized AuNPs, SEM and AFM micrographs of the synthesized nanoplates, effect of the irradiation on the synthesized AuNPs, and results of the bulk synthesis and numerical simulations (PDF)

\section{AUTHOR INFORMATION}

\section{Corresponding Author}

István Lagzi - Department of Physics, Budapest University of Technology and Economics, 1111 Budapest, Hungary; MTABME Condensed Matter Physics Research Group, Budapest University of Technology and Economics, 1111 Budapest,

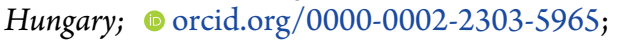

Email: istvanlagzi@gmail.com

\section{Authors}

Szabolcs Farkas - Department of Physics, Budapest University of Technology and Economics, 1111 Budapest, Hungary

Gábor Holló - MTA-BME Condensed Matter Physics Research Group, Budapest University of Technology and Economics, 1111 Budapest, Hungary

Gábor Schuszter - Department of Physical Chemistry and Materials Science, University of Szeged, 6720 Szeged, Hungary; (1) orcid.org/0000-0002-9170-9933

Ágota Deák - Department of Physical Chemistry and Materials Science, University of Szeged, 6720 Szeged, Hungary

László Janovák - Department of Physical Chemistry and Materials Science, University of Szeged, 6720 Szeged, Hungary; (1) orcid.org/0000-0002-2066-319X

Viktória Hornok - Department of Physical Chemistry and Materials Science, University of Szeged, 6720 Szeged, Hungary

Masaki Itatani - Department of Material and Biological Chemistry, Graduate School of Science and Engineering, Yamagata University, Yamagata 990-8560, Japan; (1) orcid.org/0000-0003-1025-0452

Hideki Nabika - Faculty of Science, Graduate School of Science and Engineering, Yamagata University, Yamagata 990-8560, Japan; (i) orcid.org/0000-0002-7780-8433

Dezsö Horváth - Department of Applied and Environmental Chemistry, University of Szeged, 6720 Szeged, Hungary; (1) orcid.org/0000-0003-3852-6879

Ágota Tóth - Department of Physical Chemistry and Materials Science, University of Szeged, 6720 Szeged, Hungary; @ orcid.org/0000-0001-8254-6354

Complete contact information is available at:

https://pubs.acs.org/10.1021/acs.jpcc.1c08404

\section{Notes}

The authors declare no competing financial interest.

\section{ACKNOWLEDGMENTS}

This work was supported by the National Research, Development and Innovation Office of Hungary (NN125752, NN125746, K131425, K132446, FK137555, and GINOP2.3.2-15-2016-00013), the UNKP-21-4, New National Excellence Program of the Ministry for Innovation and Technology from the source of the National Research, Development and Innovation Fund, the János Bolyai Research Scholarship of the Hungarian Academy of Sciences, and the NRDI Fund (TKP2020 IES, Grant BME-IE-NAT) based on the charter of bolster issued by the NRDI Office under the auspices of the Ministry for Innovation and Technology.

\section{REFERENCES}

(1) Schmid, G.; Corain, B. Nanoparticulated Gold: Syntheses, Structures, Electronics, and Reactivities. Eur. J. Inorg. Chem. 2003, 2003 (17), 3081-3098.

(2) Amendola, V.; Pilot, R.; Frasconi, M.; Marago, O. M.; Iati, M. A. Surface Plasmon Resonance in Gold Nanoparticles: A Review. J. Phys.: Condens. Matter 2017, 29 (20), 203002.

(3) Nakanishi, H.; Bishop, K. J. M.; Kowalczyk, B.; Nitzan, A.; Weiss, E. A.; Tretiakov, K. V.; Apodaca, M. M.; Klajn, R.; Stoddart, J. F.; Grzybowski, B. A. Photoconductance and Inverse Photoconductance in Films of Functionalized Metal Nanoparticles. Nature 2009, 460 (7253), 371-375. 
(4) Akbarzadeh, A.; Samiei, M.; Davaran, S. Magnetic Nanoparticles: Preparation, Physical Properties, and Applications in Biomedicine. Nanoscale Res. Lett. 2012, 7 (1), 144.

(5) Slepička, P.; SlepičkováKasálková, N.; Siegel, J.; Kolská, Z.; Svorčík, V. Methods of Gold and Silver Nanoparticles Preparation. Materials 2020, 13 (1), 1.

(6) Thompson, D. T. Using Gold Nanoparticles for Catalysis. Nano Today 2007, 2 (4), 40-43.

(7) Corma, A.; Garcia, H. Supported Gold Nanoparticles as Catalysts for Organic Reactions. Chem. Soc. Rev. 2008, 37 (9), 2096-2126.

(8) Primo, A.; Marino, T.; Corma, A.; Molinari, R.; García, H. Efficient Visible-Light Photocatalytic Water Splitting by Minute Amounts of Gold Supported on Nanoparticulate $\mathrm{CeO}_{2}$ Obtained by a Biopolymer Templating Method. J. Am. Chem. Soc. 2011, 133 (18), 6930-6933.

(9) Han, G.; Ghosh, P.; Rotello, V. M. Functionalized Gold Nanoparticles for Drug Delivery. Nanomedicine 2007, 2 (1), 113-123.

(10) Yeh, Y.-C.; Creran, B.; Rotello, V. M. Gold Nanoparticles: Preparation, Properties, and Applications in Bionanotechnology. Nanoscale 2012, 4 (6), 1871-1880.

(11) Saha, K.; Agasti, S. S.; Kim, C.; Li, X.; Rotello, V. M. Gold Nanoparticles in Chemical and Biological Sensing. Chem. Rev. 2012, 112 (5), 2739-2779.

(12) Elahi, N.; Kamali, M.; Baghersad, M. H. Recent Biomedical Applications of Gold Nanoparticles: A Review. Talanta 2018, 184, 537-556.

(13) Fan, J.; Cheng, Y.; Sun, M. Functionalized Gold Nanoparticles: Synthesis, Properties and Biomedical Applications. Chem. Rec. 2020, 20 (12), 1474-1504.

(14) Homberger, M.; Simon, U. On the Application Potential of Gold Nanoparticles in Nanoelectronics and Biomedicine. Philos. Trans. R. Soc., A 2010, 368 (1915), 1405-1453.

(15) Zhang, Y.; Pluchery, O.; Caillard, L.; Lamic-Humblot, A.-F.; Casale, S.; Chabal, Y. J.; Salmeron, M. Sensing the Charge State of Single Gold Nanoparticles via Work Function Measurements. Nano Lett. 2015, 15 (1), 51-55.

(16) Teranishi, T. Fabrication and Electronic Properties of Gold Nanoparticle Superlattices. C. R. Chim. 2003, 6 (8), 979-987.

(17) Jana, N. R.; Gearheart, L.; Murphy, C. J. Seeding Growth for Size Control of 5-40 Nm Diameter Gold Nanoparticles. Langmuir 2001, 17 (22), 6782-6786.

(18) Szabó, R.; Lente, G. Full Analytical Solution of a NucleationGrowth Type Kinetic Model of Nanoparticle Formation. J. Math. Chem. 2019, 57 (2), 616-631.

(19) Szabó, R.; Lente, G. A Comparison of the Stochastic and Deterministic Approaches in a Nucleation-Growth Type Model of Nanoparticle Formation. Chem. Mater. 2021, 33 (13), 5430-5436.

(20) Phan, C. M.; Nguyen, H. M. Role of Capping Agent in Wet Synthesis of Nanoparticles. J. Phys. Chem. A 2017, 121 (17), 32133219.

(21) Turkevich, J.; Stevenson, P. C.; Hillier, J. A Study of the Nucleation and Growth Processes in the Synthesis of Colloidal Gold. Discuss. Faraday Soc. 1951, 11 (0), 55-75.

(22) Xia, Y.; Xiong, Y.; Lim, B.; Skrabalak, S. E. Shape-Controlled Synthesis of Metal Nanocrystals: Simple Chemistry Meets Complex Physics? Angew. Chem., Int. Ed. 2009, 48 (1), 60-103.

(23) Sun, Y.; Xia, Y. Shape-Controlled Synthesis of Gold and Silver Nanoparticles. Science 2002, 298 (5601), 2176.

(24) Liu, Y.; Yang, L.; Shen, Y. Hydrothermal Synthesis of Gold Nanoplates and Their Structure-Dependent LSPR Properties. J. Mater. Res. 2018, 33 (18), 2671-2679.

(25) Niu, W.; Chua, Y. A. A.; Zhang, W.; Huang, H.; Lu, X. Highly Symmetric Gold Nanostars: Crystallographic Control and SurfaceEnhanced Raman Scattering Property. J. Am. Chem. Soc. 2015, 137 (33), 10460-10463.

(26) Seo, D.; Yoo, C. I.; Chung, I. S.; Park, S. M.; Ryu, S.; Song, H. Shape Adjustment between Multiply Twinned and Single-Crystalline
Polyhedral Gold Nanocrystals: Decahedra, Icosahedra, and Truncated Tetrahedra. J. Phys. Chem. C 2008, 112 (7), 2469-2475.

(27) Kuttner, C.; Mayer, M.; Dulle, M.; Moscoso, A.; LópezRomero, J. M.; Förster, S.; Fery, A.; Pérez-Juste, J.; Contreras-Cáceres, R. Seeded Growth Synthesis of Gold Nanotriangles: Size Control, SAXS Analysis, and SERS Performance. ACS Appl. Mater. Interfaces 2018, 10 (13), 11152-11163.

(28) Guo, Z.; Zhang, Y.; DuanMu, Y.; Xu, L.; Xie, S.; Gu, N. Facile Synthesis of Micrometer-Sized Gold Nanoplates through an AnilineAssisted Route in Ethylene Glycol Solution. Colloids Surf., A 2006, 278 (1), 33-38.

(29) Qin, F.; Zhao, T.; Jiang, R.; Jiang, N.; Ruan, Q.; Wang, J.; Sun, L.-D.; Yan, C.-H.; Lin, H.-Q. Thickness Control Produces Gold Nanoplates with Their Plasmon in the Visible and Near-Infrared Regions. Adv. Opt. Mater. 2016, 4 (1), 76-85.

(30) Wang, C.; Kan, C.; Zhu, J.; Zeng, X.; Wang, X.; Li, H.; Shi, D. Synthesis of High-Yield Gold Nanoplates: Fast Growth Assistant with Binary Surfactants. J. Nanomater. 2010, 2010, 969030.

(31) Ye, S.; Connell, S. D.; McLaughlan, J. R.; Roach, L.; Aslam, Z.; Chankhunthod, N.; Brown, A. P.; Brydson, R.; Bushby, R. J.; Critchley, K.; et al. One-Step Preparation of Biocompatible Gold Nanoplates with Controlled Thickness and Adjustable Optical Properties for Plasmon-Based Applications. Adv. Funct. Mater. 2020, 30 (40), 2003512.

(32) Jana, N. R.; Gearheart, L.; Murphy, C. J. Wet Chemical Synthesis of High Aspect Ratio Cylindrical Gold Nanorods. J. Phys. Chem. B 2001, 105 (19), 4065-4067.

(33) Piella, J.; Bastús, N. G.; Puntes, V. Size-Controlled Synthesis of Sub-10-Nanometer Citrate-Stabilized Gold Nanoparticles and Related Optical Properties. Chem. Mater. 2016, 28 (4), 1066-1075.

(34) Millstone, J. E.; Métraux, G. S.; Mirkin, C. A. Controlling the Edge Length of Gold Nanoprisms via a Seed-Mediated Approach. Adv. Funct. Mater. 2006, 16 (9), 1209-1214.

(35) Scarabelli, L.; Coronado-Puchau, M.; Giner-Casares, J. J.; Langer, J.; Liz-Marzán, L. M. Monodisperse Gold Nanotriangles: Size Control, Large-Scale Self-Assembly, and Performance in SurfaceEnhanced Raman Scattering. ACS Nano 2014, 8 (6), 5833-5842.

(36) Miranda, A.; Malheiro, E.; Skiba, E.; Quaresma, P.; Carvalho, P. A.; Eaton, P.; de Castro, B.; Shelnutt, J. A.; Pereira, E. One-Pot Synthesis of Triangular Gold Nanoplates Allowing Broad and Fine Tuning of Edge Length. Nanoscale 2010, 2 (10), 2209-2216.

(37) Pienpinijtham, P.; Han, X. X.; Suzuki, T.; Thammacharoen, C.; Ekgasit, S.; Ozaki, Y. Micrometer-Sized Gold Nanoplates: StarchMediated Photochemical Reduction Synthesis and Possibility of Application to Tip-Enhanced Raman Scattering (TERS). Phys. Chem. Chem. Phys. 2012, 14 (27), 9636-9641.

(38) Nabika, H.; Itatani, M.; Lagzi, I. Pattern Formation in Precipitation Reactions: The Liesegang Phenomenon. Langmuir 2020, 36 (2), 481-497.

(39) Szücs, R.; Balogh-Weiser, D.; Sánta-Bell, E.; Tóth-Szeles, E.; Varga, T.; Kónya, Z.; Poppe, L.; Lagzi, I. Green Synthesis and in Situ Immobilization of Gold Nanoparticles and Their Application for the Reduction of P-Nitrophenol in Continuous-Flow Mode. RSC Adv. 2019, 9 (16), 9193-9197.

(40) Zakhia Douaihy, R.; Al-Ghoul, M.; Hmadeh, M. Liesegang Banding for Controlled Size and Growth of Zeolitic-Imidazolate Frameworks. Small 2019, 15 (28), 1901605.

(41) Kalash, L.; Farah, H.; Eddin, A. Z.; Sultan, R. Dynamical Profiles of the Reactive Components in Direct and Revert Liesegang Patterns. Chem. Phys. Lett. 2013, 590, 69-73.

(42) Kanniah, N.; Gnanam, F. D.; Ramasamy, P.; Laddha, G. S. Revert and Direct Type Liesegang Phenomenon of Silver Iodide. J. Colloid Interface Sci. 1981, 80 (2), 369-376.

(43) Kanniah, N.; Gnanam, F. D.; Ramasamy, P. Revert and Direct Liesegang Phenomenon of Silver Iodide: Factors Influencing the Transition Point. J. Colloid Interface Sci. 1983, 94 (2), 412-420.

(44) Karam, T.; El-Rassy, H.; Sultan, R. Mechanism of Revert Spacing in a PbCrO4 Liesegang System. J. Phys. Chem. A 2011, 115 (14), 2994-2998. 
(45) Walliser, R. M.; Boudoire, F.; Orosz, E.; Tóth, R.; Braun, A.; Constable, E. C.; Rácz, Z.; Lagzi, I. Growth of Nanoparticles and Microparticles by Controlled Reaction-Diffusion Processes. Langmuir 2015, 31 (5), 1828-1834.

(46) Saliba, D.; Ammar, M.; Rammal, M.; Al-Ghoul, M.; Hmadeh, M. Crystal Growth of ZIF-8, ZIF-67, and Their Mixed-Metal Derivatives. J. Am. Chem. Soc. 2018, 140 (5), 1812-1823.

(47) Kumar, S.; Gandhi, K. S.; Kumar, R. Modeling of Formation of Gold Nanoparticles by Citrate Method. Ind. Eng. Chem. Res. 2007, 46 (10), 3128-3136.

(48) Bastús, N. G.; Comenge, J.; Puntes, V. Kinetically Controlled Seeded Growth Synthesis of Citrate-Stabilized Gold Nanoparticles of up to $200 \mathrm{Nm}$ : Size Focusing versus Ostwald Ripening. Langmuir 2011, 27 (17), 11098-11105.

(49) Shi, L.; Buhler, E.; Boué, F.; Carn, F. How Does the Size of Gold Nanoparticles Depend on Citrate to Gold Ratio in Turkevich Synthesis? Final Answer to a Debated Question. J. Colloid Interface Sci. 2017, 492, 191-198.

(50) Rahbani, J.; Behzad, A. R.; Khashab, N. M.; Al-Ghoul, M. Characterization of Internal Structure of Hydrated Agar and Gelatin Matrices by Cryo-SEM. Electrophoresis 2013, 34 (3), 405-408.

(51) Geim, A. K.; Novoselov, K. S. The Rise of Graphene. Nat. Mater. 2007, 6 (3), 183-191. 